\title{
Importin-as are required for the nuclear localization and function of the Plasmopara viticola effector PvAVH53
}

Tingting Chen ${ }^{1,2,3}$, Jing Peng ${ }^{1,2,3}$, Xiao Yin ${ }^{1,2,3}$, Meijie Li ${ }^{1,2,3}$, Gaoqing Xiang ${ }^{1,2,3}$, Yuejin Wang $\mathbb{1}^{1,2,3}$, Yan Lei ${ }^{4}$ and $\operatorname{Yan} \mathrm{Xu}^{1,2,3}$

\begin{abstract}
Plant pathogenic oomycetes deliver a troop of effector proteins into the nucleus of host cells to manipulate plant cellular immunity and promote colonization. Recently, researchers have focused on identifying how effectors are transferred into the host cell nucleus, as well as the identity of the nuclear targets. In this study, we found that the RxLR effector PvAVH53 from the grapevine (Vitis vinifera) oomycete pathogen Plasmopara viticola physically interacts with grapevine nuclear import factor importin alphas (VvImpa and VvImpa4), localizes to the nucleus and triggers cell death when transiently expressed in tobacco (Nicotiana benthamiana) cells. Deletion of a nuclear localization signal (NLS) sequence from PVAVH53 or addition of a nuclear export signal (NES) sequence disrupted the nuclear localization of PVAVH53 and attenuated its ability to trigger cell death. Suppression of two tobacco importin-a genes, namely, Nblmp-a1 and Nblmp-a2, by virus-induced gene silencing (VIGS) also disrupted the nuclear localization and ability of PVAVH53 to induce cell death. Likewise, we transiently silenced the expression of VvImpa/a4 in grape through CRISPR/ Cas13a, which has been reported to target RNA in vivo. Finally, we found that attenuating the expression of the Importin-as genes resulted in increased susceptibility to the oomycete pathogen Phytophthora capsici in N. benthamiana and $P$. viticola in $V$. vinifera. Our results demonstrate that importin-as are required for the nuclear localization and function of PVAVH53 and are essential for host innate immunity. The findings provide insight into the functions of importin-as in grapevine against downy mildew.
\end{abstract}

\section{Introduction}

Pathogenic oomycetes are responsible for some of the most widespread and devastating diseases of crop and ornamental plants. These diseases include potato late blight, caused by Phytophthora infestans, and grapevine downy mildew, caused by Plasmopara viticola. Similar to many other plant pathogens, oomycetes secrete a troop of effector molecules inside host cells to promote pathogenicity ${ }^{1-4}$. Many effectors, including RxLR proteins, have

\footnotetext{
Correspondence: Yan Xu (yan.xu@nwsuaf.edu.cn)

${ }^{1}$ State Key Laboratory of Crop Stress Biology in Arid Areas (Northwest A\&F University), Yangling, Shaanxi, P.R. China

${ }^{2}$ College of Horticulture, Northwest A\&F University, Yangling, Shaanxi, P.R. China

Full list of author information is available at the end of the article
}

been shown to localize to the host cell nucleus and suppress the expression of genes that promote innate immune responses ${ }^{5,6}$. For example, a majority of the RxLR effectors studied from the oomycete pathogen Hyaloperonospora arabidopsidis target the plant cell nucleus ${ }^{6}$. The RxLR effectors Pi04089 and Pi04314 from P. infestans localize to the potato host cell nucleus; Pi04089 targets the K-homology $(\mathrm{KH})$ RNA-binding protein (StKRBP1) to promote colonization, while Pi04314 enhances colonization by attenuating the induction of jasmonic acid- and salicylic acid-related immune responses ${ }^{7,8}$. Another $P$. infestans RxLR effector, PITG_22798, is transferred to the nucleus to induce cell death in tobacco ${ }^{9}$. In addition, activation of the innate immune response by the resistance protein $\mathrm{R} 1$ requires colocalization of the RxLR

\section{(c) The Author(s) 2021}

(c) (i) Open Access This article is licensed under a Creative Commons Attribution 4.0 International License, which permits use, sharing, adaptation, distribution and reproduction c. in any medium or format, as long as you give appropriate credit to the original author(s) and the source, provide a link to the Creative Commons license, and indicate if changes were made. The images or other third party material in this article are included in the article's Creative Commons license, unless indicated otherwise in a credit line to the material. If material is not included in the article's Creative Commons license and your intended use is not permitted by statutory regulation or exceeds the permitted use, you will need to obtain permission directly from the copyright holder. To view a copy of this license, visit http://creativecommons.org/licenses/by/4.0/. 
effector AVR $1^{10}$. Although these studies underscore the importance of the nuclear localization of effector proteins in modulating plant defense, there have been few insights into the associated mechanism(s).

The nuclear envelope is highly selective for the dynamic exchange of macromolecules between the nucleus and cytoplasm $^{11-14}$. The classic nuclear import pathway for proteins depends on the binding of a short nuclear localization signal (NLS) sequence by importin- $\alpha$, interaction with importin-â, and shuttling of the protein through the nuclear pore complex ${ }^{15,16}$. Importin- $\alpha$ proteins typically contain three conserved domains: an amino-terminal importin-â-binding domain (IBB), tandem armadillo (Arm) repeats that interact with the NLS region of the cargo protein, and a carboxyl-terminal region that binds a nuclear export factor to facilitate its return to the cytoplasm $^{17,18}$. Canonical NLSs are classified as monopartite NLSs, with a single cluster of basic amino residues (K[K/ $\mathrm{R}] \mathrm{X}] \mathrm{K} / \mathrm{R}]$ ), and bipartite NLSs, containing two clusters $\left.\left([\mathrm{K} / \mathrm{R}][\mathrm{K} / \mathrm{R}] \mathrm{X}_{10-12}\right][\mathrm{K} / \mathrm{R}]_{3 / 5}\right)$ bridged by linker amino acids $^{19,20}$.

Increasing evidence suggests that the nuclear localization of RxLR effectors is dependent on NLS-importin pathways. For example, the ability of CRN8 and CRN108 from P. infestans and PvRxLR16 from P. viticola to induce plant cell death is dependent on their nuclear localization $^{21-23}$. The nuclear-targeted effector HaRxL106 from $H$. arabidopsidis was reported to interact with importin$\alpha^{24}$. In Nicotiana benthamiana, silencing of the importin$\alpha$ genes NbIMP $\alpha 1$ and NbIMP $\alpha 2$ via virus-induced gene silencing (VIGS) disrupted nuclear localization of the $P$. infestans effectors Nuk6 and Nuk $7^{12}$ as well as the SAP11 effector from the phytoplasma Candidatus Phytoplasma asteris $^{25}$. Furthermore, an engineered SAP11 protein in which the NLS was disrupted and a nuclear exclusion sequence was appended to the carboxyl terminus failed to target the nucleus and was associated with weakened pathogenicity ${ }^{26}$.

Several recent studies have also suggested that nuclear localization of pathogen effectors is required for triggering the plant innate immune response. For example, in Arabidopsis, the leucine-rich-repeat (LRR) innate immune receptor TN13 physically interacts with the importin- $\alpha$ MOS6/Imp- $\alpha 3$, and mos 6 mutants showed enhanced susceptibility to the oomycete Hyaloperonospora parasitica $^{27,28}$. The RxLR effector PvAvh74 from P. viticola also requires nuclear localization to induce immune responses in tobacco ${ }^{29}$. Thus, interactions between importin- $\alpha$ and both effectors and innate immunityrelated proteins play important roles in plant defense responses against pathogens ${ }^{12,27,30-32}$.

In previous preliminary work, we observed that the $P$. viticola effector PvAVH53 localized to the nucleus and triggered cell death in N. benthamiana ${ }^{33}$. In this study, we report that PvAVH53 physically interacts with importinas (VvImp $\alpha / \mathrm{VvImp} \alpha 4$, and NbImp $\alpha 1 / \mathrm{NbImp} \alpha 2)$ from $V$. vinifera and $N$. benthamiana and that these interactions are crucial for the function of the effector PvAVH53. Engineered PvAVH53 proteins lacking the NLS or containing a nuclear export signal (NES) localized to both the nucleus and cytoplasm of $N$. benthamiana cells and showed significantly attenuated activity to trigger cell death. In addition, both the nuclear localization and cell death activity of PvAVH53 were repressed in tobacco cells in which the expression of two VvImpa4 homologs, namely, NbImp $\alpha 1$ and NbImp $\alpha 2$, was repressed. Likewise, we transiently silenced the expression of $\mathrm{VvImp} \alpha / \alpha 4$ in grape through CRISPR/Cas13a, which has been reported to target RNA in vivo. Finally, attenuating the expression of the Importin- $\alpha$ s genes resulted in increased susceptibility to the oomycete pathogen Phytophthora capsici in $N$. benthamiana and $P$. viticola in $V$. vinifera. Taken together, these investigations demonstrate that importin$\alpha$ is required for the nuclear localization and function of PvAVH53. They provide a solid foundation for advanced study of importin- $\alpha$ s in grapevine against downy mildew.

\section{Results}

Nuclear localization of PvAVH53 is essential for triggering cell death

Previously, we identified several $P$. viticola RxLR effectors capable of triggering cell death when expressed transiently in $N$. benthamiana cells. Our preliminary results suggested that among these effectors, PvAVH53 and PvAVH54804 induced cell death the most rapidly and at the lowest apparent concentration ${ }^{33}$. Imaging of $N$. benthamiana protoplasts transiently expressing PvAVH53-GFP or PvAVH54804-GFP showed that PvAVH53-GFP localizes to the nucleus, whereas PvAVH54804-GFP accumulates in both the nucleus and cytoplasm (Fig. 1a, c). To determine whether nuclear localization is required for PvAVH53mediated induction of cell death, a NES sequence was appended to the carboxyl terminus of PvAVH53 $\left(\right.$ PvAVH53 ${ }^{\text {NES }}$ ), or the NLS peptide sequence was deleted from PvAVH53 (PvAVH53 ${ }^{\Delta N L S}$ ), and these modified PvAVH53s were expressed in tobacco leaf protoplasts. In both cases, a substantial increase in fluorescence was observed outside the nucleus, indicating substantial interference with nuclear accumulation of PvAVH53 (Fig. 1a). We observed similar results when expression was evaluated in $V$. vinifera protoplasts (Fig. S1a). We also modified PvAVH54804 by the addition of a C-terminal NES or NLS peptide (PvAVH54804 ${ }^{\mathrm{NES}}$, PvAVH54804 ${ }^{\mathrm{NLS}}$, respectively). In contrast to the modified PvAVH53, the PvAVH54804 ${ }^{\text {NES }}$ and PvAVH54804 ${ }^{\mathrm{NLS}}$ proteins showed essentially complete targeting to the nucleus or cytoplasm in $V$. vinifera and $N$. benthamiana leaf cells (Fig. S1b; Fig. 1b). To determine whether a change in subcellular localization affected the 

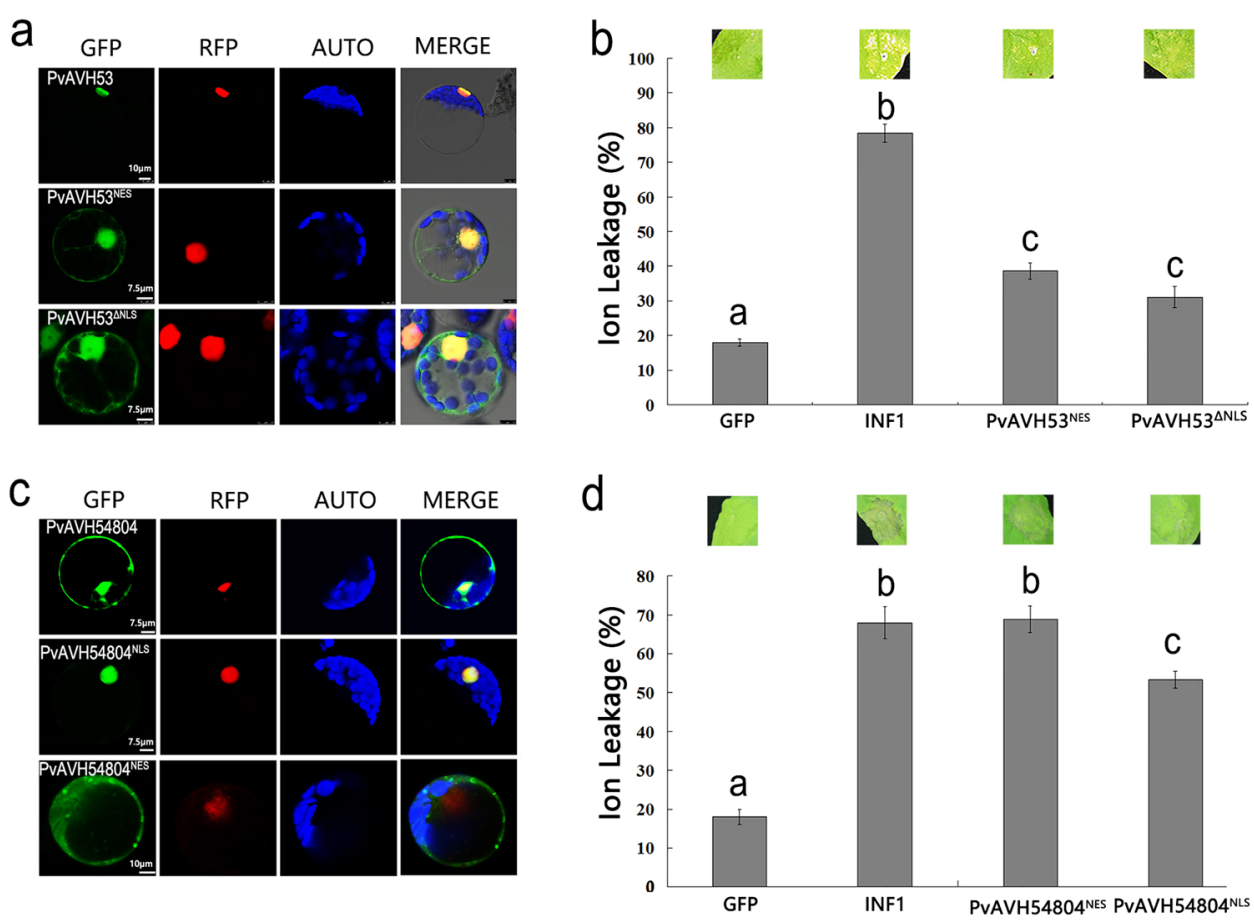

Fig. 1 PvAVH53 requires nuclear localization to trigger cell death but PvAVH54804 does not. a Transient expression of PvAVH53 with a nuclear export signal (PVAVH53 ${ }^{\mathrm{NES}}$ ) or nuclear localization signal (NLS) deletion (PVAVH53 ${ }^{\triangle N L S}$ ) cotransformed with an NLS-mCherry marker in Nicotiana benthamiana protoplasts, showing that both mutants of PVAVH53 failed to stabilize nuclear localization. $\mathbf{b}$ Transient expression of the PVAVH53 mutants in N. benthamiana leaves by agroinfiltration showed that PVAVH53 mutants failed to trigger cell death. $\mathbf{c}$ Transient expression of PVAVH54804 with a nuclear export signal (NES) or nuclear localization signal (NLS) cotransformed with an NLS-mCherry marker in N. benthamiana protoplasts. d Transient expression of the PVAVH54804 mutants in N. benthamiana leaves by agroinfiltration showed that PVAVH54804 ${ }^{\text {NLS }}$ failed to trigger cell death. Photos were captured at $6 \mathrm{~d}$ post infiltration. Ion leakage from the infiltrated leaf discs was measured as a percentage of leakage from boiled discs to quantify cell death. Scale bar $=5-10 \mu \mathrm{m}$. The data are the means \pm SEs based on three independent replicates (Student's $t$-test, $P<0.01)$

function of the effectors, the effector-GFP fusion proteins were transiently expressed in $N$. benthamiana leaf cells using agroinfiltration, and ion leakage was measured $4 \mathrm{~d}$ after infiltration ${ }^{34}$. We found that PvAVH53 ${ }^{\mathrm{NES}}$ showed a strongly reduced ability to induce cell death compared with nonmodified PvAVH53 (Fig. 1b). We also found that PvAVH54804 ${ }^{\text {NES }}$ triggered cell death to a similar degree as nonmodified PvAVH54804, while PvAVH54804 ${ }^{\mathrm{NLS}}$ showed a significantly decreased ability to induce cell death (Fig. 1d). Taken together, these findings demonstrated that nuclear localization of PvAVH53 is required for full activity to induce cell death, whereas PvAVH54804 mostly requires cytoplasmic localization to induce plant cell death.

\section{PvAVH53 interacts with the importin VvIMPa/a4}

We previously found that PvAVH53 localizes to the nucleus when expressed transiently in $N$. benthamiana cells $^{33}$. To identify potential interaction partners of PvAVH53 in $V$. vinifera, we conducted a yeast two-hybrid assay using PvAVH53 as bait in combination with a $V$. vinifera cDNA expression library. This resulted in the identification of multiple clones representing two distinct genes, both previously annotated as importin subunit alpha, namely, VvImp $\alpha$ and VvImp $\alpha$, through an NCBI BLAST search. To evaluate the interaction between PvAVH53 and VvImp $\alpha / \alpha 4$, we cloned a cDNA from $V$. vinifera encoding the full-length, 1,608-bp VvIMP $\alpha 4$ and 1590-bp VvIMPa open reading frames (ORFs) into the prey vector and a cDNA encoding PvAVH53 into the bait vector. This $\mathrm{Y} 2 \mathrm{H}$ assay showed that the two proteins interact in yeast cells (Fig. 2). VvImpa4 contains an amino-terminal importin beta-binding (IBB) domain (amino acids 100-200) and nine armadillo (Arm)/betacatenin-like repeats (Fig. 2). A short, amino-terminal segment of the VvImpa4 protein containing the IBB domain showed no interaction with PvAVH53 (Fig. 2). An internal segment of VvImpa4 lacking both the $\mathrm{N}$ terminus and C-terminus and expressing only eight of the nine Arm domains showed a weak interaction with PvAVH53 (Fig. 2). Interestingly, a short segment of VvImpa4 containing only the C-terminal Arm domain showed a strong interaction with PvAVH53 (Fig. 2). 


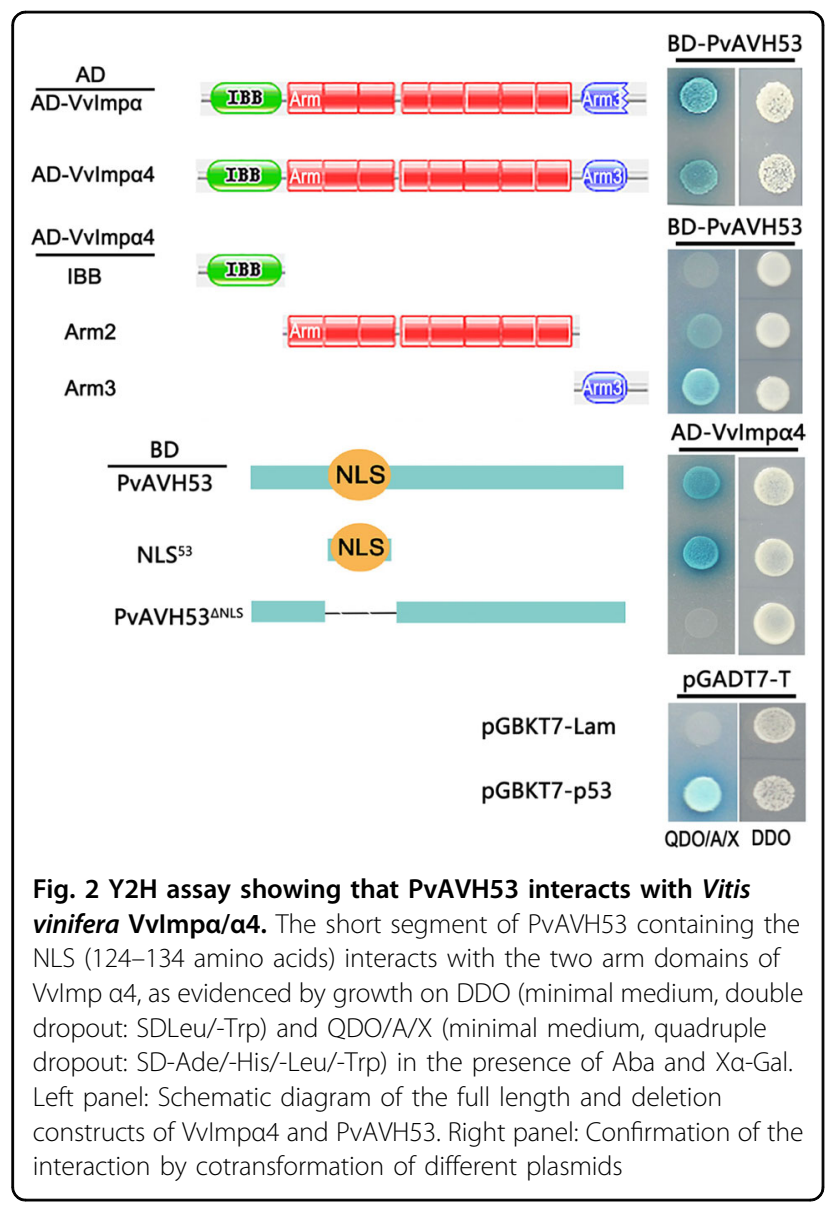

We also engineered deletion mutants of PvAVH53 to identify specific regions of the PvAVH53 protein important for interaction with VvImpa4. We found that a short segment of PvAVH53 containing the NLS was both required and sufficient for interaction with VvImpa4 (Fig. 2). We also used this two-hybrid assay to evaluate the interaction between PvAVH53 and VvImpa. Similar to VvImpa4, a strong interaction was observed when a full-length VvImp$\alpha$ clone was used as bait (Fig. 3a). Finally, we used the yeast two-hybrid assay to evaluate the interaction between VvImpa4 and a panel of 10 P. viticola RxLR-type effectors ${ }^{33}$ previously identified in our laboratory (the details of the effectors are provided in the supplementary material in Table S1). With two exceptions (PvAVH1 and PvAVH54804), all effectors showed interactions with VvImpa4 (Fig. S2). These results suggest that grapevine importin- $\alpha$ proteins may participate in the nuclear import of a wide range of RxLR effectors.

\section{PvAVH53 and VvImpas localize to and interact in the nucleus}

To further document the interaction between PvAVH53 and VvImpas, we transformed $V$. vinifera protoplasts with clones expressing the proteins fused with GFP and examined their intracellular localization via fluorescence microscopy. An NLS-mCherry marker was coexpressed to visualize the nucleus. This revealed that both PvAVH53GFP and VvImpa-GFP fusion proteins localized to the nucleus (Fig. 3a). We further analyzed the interaction using bimolecular fluorescence complementation (BiFC). PvAVH53 carrying a carboxyl-terminal fragment of YFP (PvAVH53-YC) was coexpressed with VvImpa or VvImp 4 with amino-terminal fusion of YFP (VvImpaYN or VvImpa4-YN, respectively) in $V$. vinifera protoplasts. YFP fluorescence was observed in the nucleus, indicating that VvImp $\alpha$ and VvImpo4 interact with PvAVH53 in the host nucleus (Fig. 3b).

In summary, these two complementary approaches provided solid evidence that the RxLR effector PvAVH53 interacts with $V$. vinifera VvImp $\alpha$ and VvImpa4.

\section{PvAVH53 interacts with Importin-as in tobacco leaf cells}

VvImp $\alpha$ and VvImpa4 show strong amino acid sequence homology (up to 86.3\%) and close phylogenetic relationships with two importin- $\alpha$ s from $N$. benthamiana, designated NbImpa1 and NbImpa2 (Fig. 4). To evaluate the interaction between PvAVH53 and these two importin- $\alpha$ s from tobacco by the yeast two-hybrid approach, we cloned cDNAs from $N$. benthamiana encoding the full-

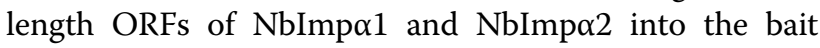
vector and PvAVH53 cDNA into the prey vector. We also used BiFC to evaluate interactions between PvAVH53 and tobacco importin- $\alpha$ s. PvAVH53-YC was coexpressed with

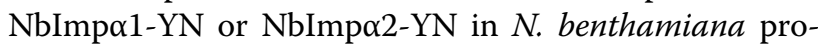
toplasts. YFP fluorescence was observed in the nucleus, indicating that NbImp $\alpha 1$ and NbImp $\alpha 2$ interact with PvAVH53 in the nucleus (Fig. S3).

\section{Silencing of Nblmpa $1 / 2$ reduces plant growth and disturbs the nuclear localization of PvAVH53}

PvAVH53, but not PvAVH54804, contains a predicted NLS at amino acids 124-134 (AA). Importin- $\alpha$ s mediate the nuclear import of various cargo proteins carrying an NLS signal. To determine whether importin- $\alpha$ s are involved in the cell death-triggering activity of PvAVH53,

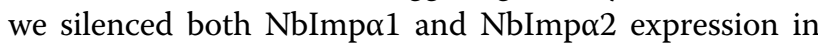
$N$. benthamiana using VIGS ${ }^{2}$. The primers were designed to amplify an $\sim 0.6 \mathrm{~kb}$ fragment of NbImpa 1 and NbImpa2, as described in Schornack et al $2010^{21}$, which was then ligated into $\mathrm{TRV}^{35}$. N. benthamiana leaves were then subjected to agroinfiltration with TRV1 and a mixture of TRV2:NbImp1ENbImpa2 and TRV2:GFP (TRV2:GFP was included as a negative control to visualize TRV infection). Primers for detection were designed for specificity with the silenced segment of NbImpa1/2. Leaves of the silenced $N$. benthamiana plants grew more slowly than those of the TRV:GFP control (Fig. 5a), and $\mathrm{RT}-\mathrm{PCR}$ results revealed that NbImpos transcript levels in 

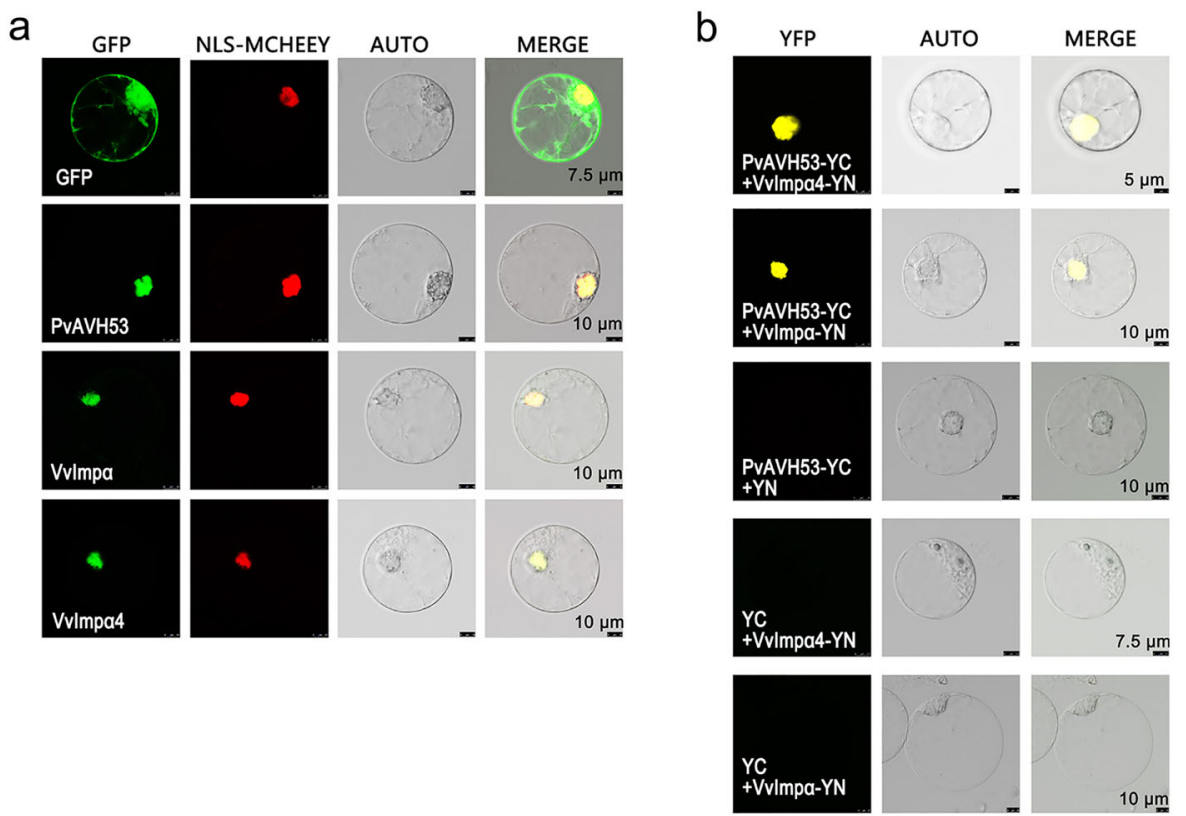

Fig. 3 PvAVH53 and VvImpas localize to and interact in the Vitis vinifera cell nucleus. a Subcellular localization of $V$. vinifera nuclear import factors VvImpa4NVImpa, PVAVH53, and GFP as controls cotransformed with NLS-mCherry (as the nuclear localization marker) in $\mathrm{V}$. vinifera protoplasts. b A BiFC assay confirmed that VvImpas interacted with PVAVH53. Photos were taken after protoplasts were incubated for 20-24 $\mathrm{h}$ under weak lighting at $25^{\circ} \mathrm{C}$. Scale bar $=5-10 \mu \mathrm{m}$

a

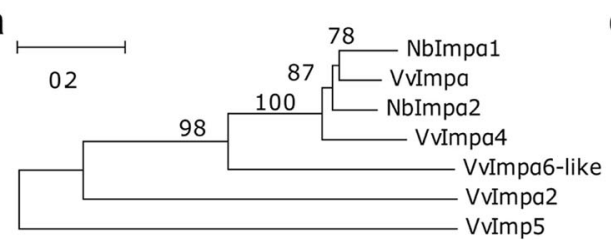

C

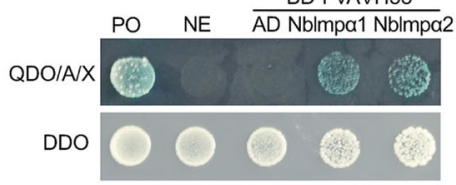

b

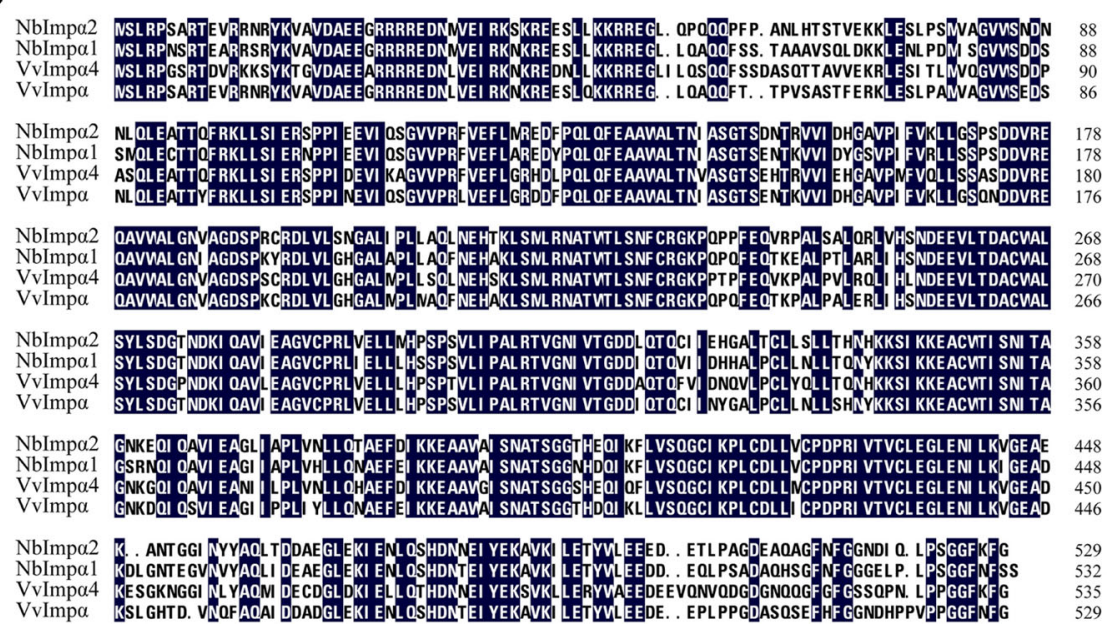

Fig. 4 PvAVH53 interacts with Nicotiana benthamiana Nblmpas. a Phylogenetic analyses of the Vvlmpas (Vvlmpa XP_002274422.1, Vvlmpa2 XP_002282816.1, Vvlmpa4 XP_002281670.1, Vvlmpa6-like XP_010646172.1, Vvlmpa5 XP_002281591.1), and Nblmpas (Nblmpa1: EF137253.1 and Nblmpa2: EF137254.1) were conducted with MEGA5 software. $\mathbf{b}$ Sequence analysis of the VvImpa/a4 and Nblmpa1/2. The sequence alignment of the Nblmpas and Vvlmpas indicated that VvImpa and Vvlmpa4 showed high sequence identity with the two Nblmpas. c Nblmpa1/2 interact with PvAVH53 in yeast Y2H Gold cells. The yeast cells grew and turned blue on DDO (SD-Leu/-Trp) and QDO/A/X (SD-Ade/-His/-Leu/-Trp) in the presence of Aba and X-a-Gal 


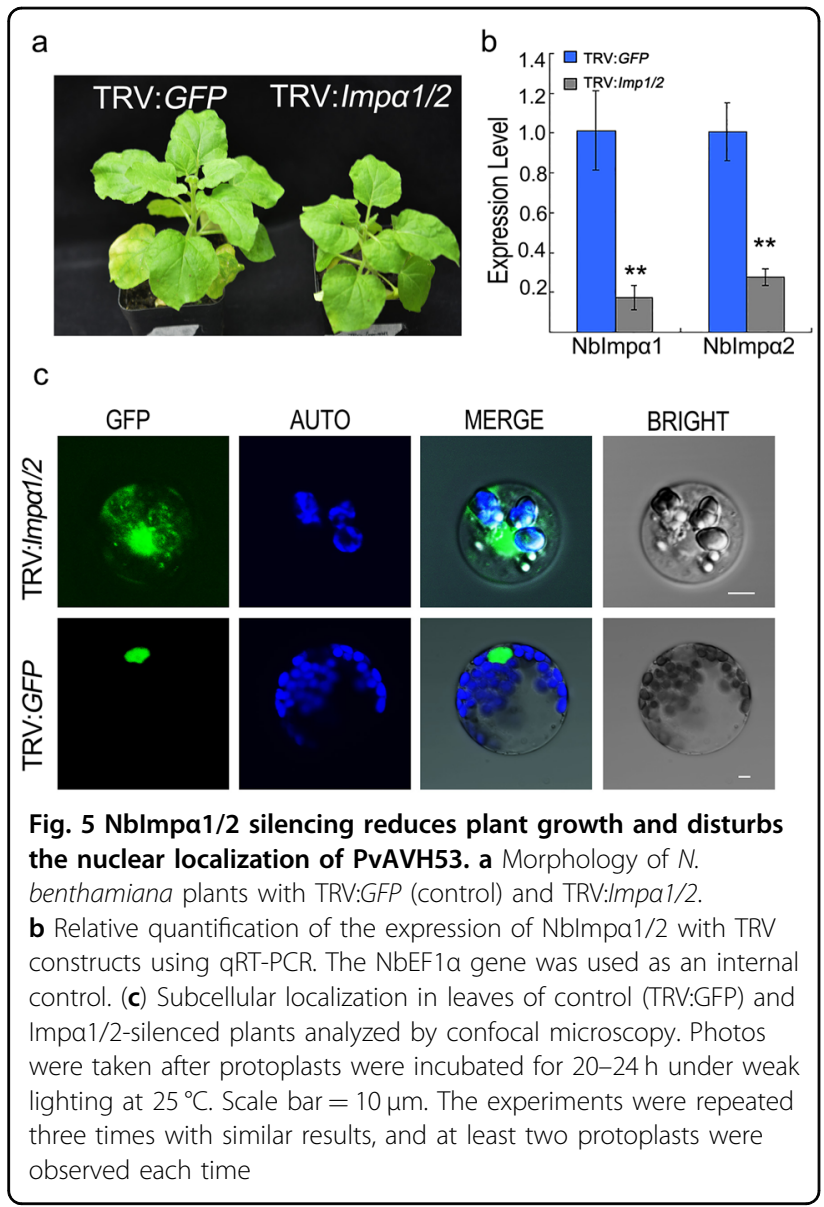

the leaves of silenced plants were significantly lower than those in the control leaves (Fig. 5b). We also isolated protoplasts from NbImp $\alpha 1 / 2$-silenced plants and introduced the GFP:PvAVH53 construct. Confocal microscopy showed that the subcellular localization of PvAVH53 in NbImp $1 / 2$-silenced protoplasts was similar to that of PvAVH53 ${ }^{\text {NES }}$ in noninfiltrated tobacco leaf cells (Fig. 5c), indicating that NbImp $1 / 2$ was successfully silenced in $N$. benthamiana and that PvAVH53 was relocated in the plant nucleus and cytoplasm.

\section{Silencing of Nblmpa $1 / 2$ diminishes cell death triggered by PvAVH53 and promotes the susceptibility of $N$. benthamiana to Phytophthora capsici}

Detection of the ion leakage of the control (TRV-GFP and WT) and NbImpo1/2-silenced tobacco leaves showed that virus-induced gene silencing (VIGS) in Nicotiana benthamiana had no significant effect on ion leakage (Fig. S4). Both PvAVH53 and PvAVH54804 were transiently expressed by agroinfiltration in NbImpo1/2-silenced and control $N$. benthamiana leaves. PvAVH54804 induced cell death in both NbImp $\alpha 1 / 2$-silenced and control leaves. In contrast, PvAVH53 showed only a weak ability to induce cell death and to promote ion leakage in Impo1/2-

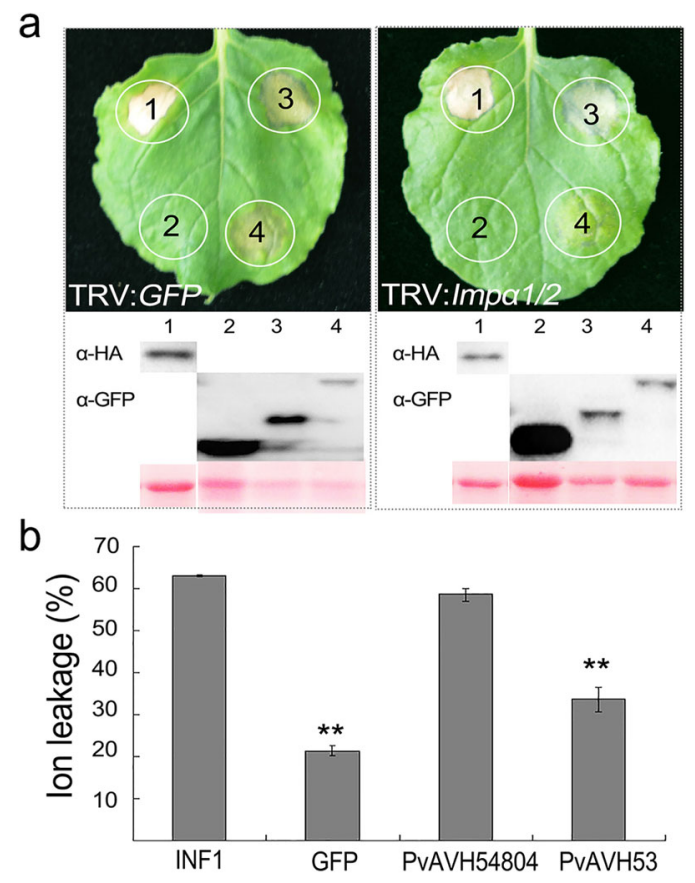

Fig. 6 Nblmpa1/2 silencing decreases the cell death triggered by PvAVH53 but PvAVH54804 does not. a Leaves of control (TRV:GFP) and Impa1/2-silenced plants were agroinfiltrated with PVAVH53/ PvAVH54804 expression plasmids. Proteins were extracted from infiltrated spots to analyze the expression (spot number: 1, INF1; 2 , GFP; 3, PVAVH54804; 4, PvAVH53). b lon leakage (\%) measurement at infiltration sites. The data are the means \pm SEs based on three independent replicates (Student's t-test: ${ }^{*} P<0.01$ )

silenced leaves compared with the control (Fig. 6a, b). Based on this finding, we concluded that the nuclear localization of PvAVH53, as well as its ability to trigger cell death, depends on NbImp $1 / 2$.

To study the role of NbImp $1 / 2$ in the defense against pathogens, we evaluated the potential of $P$. capsici to infect leaves from NbImpa1/2-silenced tobacco plants. We found that the lesions on NbImpa1/2-silenced tobacco plants were larger than the lesions on control plants (Fig. S5a). Measurement of the lesion area of $P$. capsici provided independent evidence that NbImpo1/2silenced tobacco plants were more susceptible to $P$. capsici (Fig. S5b).

\section{Silencing of Vvlmpa/a4 disorders the sublocalization of PvAVH53 and promotes the susceptibility of $V$. vinifera to P. viticola}

To demonstrate the roles of VvImp as in the nuclear localization of PvAVH53 in grape and in the defense against $P$. viticola, we transiently silenced the expression of VvImp $\alpha / \alpha 4$ in grape through CRISPR/Cas13a, which has been reported to target RNA in vivo ${ }^{36,37}$. Transient expression of PvAVH53 in grape leaves cotransformed with pCR11 or pCR11-VvImp $/ \alpha 4$ showed that PvAVH53 
a

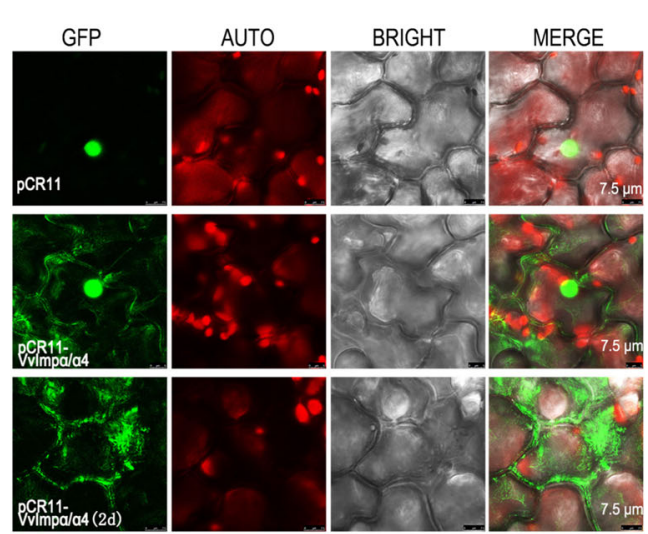

C

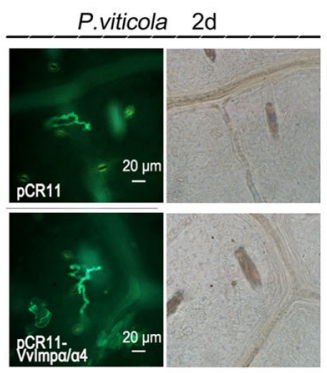

b

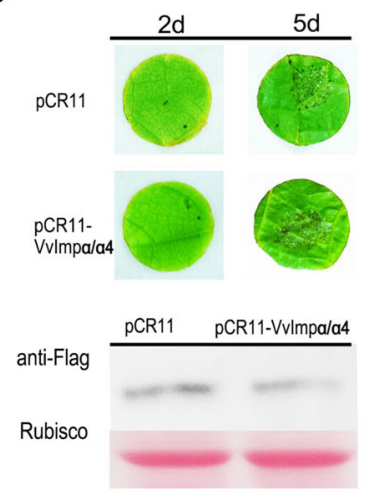

d

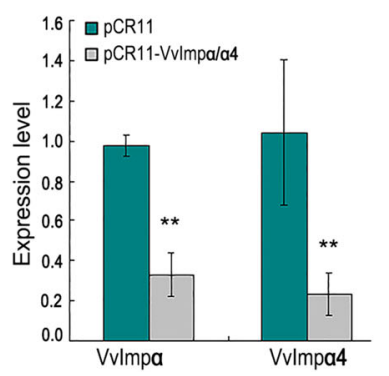

Fig. 7 VvImpa/a4 silencing changes the sublocalization of PvAVH53 and promotes the susceptibility of $V$. vinifera to $P$. viticola. a Subcellular localization of PVAVH53 on leaves of control (pCR11) and pCR11-VVImpa/a4-silenced grape leaves was analyzed by confocal microscopy. Scale bar $=$ 5-7.5 $\mathrm{mm}$. b Phenotype of control ( $\mathrm{PCR} 11)$ and pCR11-VvImpa/a4-silenced grape leaves infected with $P$. viticola. Leaf discs were photographed at 2 days and 5 days post infection. Western blotting detected Cas13a protein expression in control and Vv/mpa/a4-silenced grape leaves. $\mathbf{c} P$. viticola development at inoculation sites in leaf discs of control and VVImpa/a4-silenced grape leaves was revealed by aniline blue staining. $\mathbf{d}$ Relative quantification of the expression of Vvlmpa/a4 in control and VvImpa/a4-silenced grape leaves via qRT-PCR. The VVActin (AY680701) gene was used as an internal reference. The data are the means \pm SEs based on three independent replicates (Student's $t$-test: $* * P<0.01$ )

also failed to target the nucleus only in the host plant. Even though PvAVH53 was expressed in grape after 2 days of infection with pCR11-VvImp $\alpha / \alpha 4$, its localization was obviously rearranged in the cytoplasm (Fig. 7a). Then, we evaluated the potential of $P$. viticola to infect leaves from control (pCR11) and pCR11-VvImpa/ $\alpha 4$-silenced grape plants. Leaf discs obtained from the grape leaves were agroinfiltrated with control (pCR11) and pCR11-VvImpa/ $\alpha 4$ expression plasmids, infected with a $P$. viticola sporangium suspension, and cultured in a greenhouse under a 16-h light $/ 8$-h dark photoperiod at $25^{\circ} \mathrm{C}$ (light) and $18{ }^{\circ} \mathrm{C}$ (dark). We found that sporangia on VvImp $\alpha / \alpha 4$-silenced grape leaves were more abundant than sporangia on control grape leaves (Fig. 7b). Western blotting showed that the Cas13a protein was expressed in control and $V v I m p \alpha 4$-silenced grape leaves, and quantitative real-time PCR revealed that VvImpo and VvImpo4 transcript levels in $V v \operatorname{Imp\alpha } / \alpha 4$-silenced leaves were significantly lower than those in the control leaves (Fig. 7b, d). To assess P. viticola development in control and $V v \operatorname{Imp} \alpha / \alpha 4$-silenced grape leaves, the infection sites were stained with aniline blue and observed by a fluorescence microscope at 2 days and 5 days post infection, respectively. Observation of infection at 2 days showed that the encysting zoospore formed a germinative tube in the control and VvImp $\alpha 4$-silenced grape leaves. At 5 days post infection, hyphae were obviously visible in the leaf tissues of the control and VvImp $\alpha 4$-silenced grape plants, but the hyphae were more abundant and sporangiophores or sporangia were apparent in $V v \operatorname{Imp} \alpha / \alpha 4$-silenced grape leaves (Fig. 7b, c).

\section{Discussion}

The success of a pathogen depends on its capacity to overcome the host plant's innate immune responses. Effectors play a significant role in the plant-pathogen battle by interrupting host cellular processes and promoting colonization. The proximity of the plant cell nucleus to the developing oomycete haustorium has been observed more than once, suggesting that the haustorium may influence the intracellular architecture of the cell for 
efficient delivery of effectors to disturb nuclear defensive responses $^{6,38,39}$. In this study, we found that the RxLRtype effector PvAVH53 from $P$. viticola interacts with VvImp $\alpha / \alpha 4$ and enters the plant nucleus through the classic nuclear import pathway and triggers cell death. To our knowledge, this is the first evidence that $P$. viticola effectors share the canonical nuclear import machinery by targeting the adaptor importin- $\alpha$, as has been shown in other oomycetes, such as Hyaloperonospora arabidopsidis and $P$. infestans.

Previous experiments have indicated that the $P$. viticola effectors PvAVH53 and PvAVH54804 can induce cell death in $N$. benthamiana and are localized strictly in the nucleus or both in the nucleus and cytoplasm, respectively ${ }^{33}$. Our experiments further showed that PvAVH53 and PvAVH54804 induce cell death in N. benthamiana. Many studies, especially in the oomycete pathogen model $P$. infestans, have investigated interactions between pathogens and plant host cells ${ }^{4,40}$, but the host targets of the P. viticola effectors and associated pathogenic mechanisms have rarely been described.

In our experiments, the addition of a nuclear export signal (NES) sequence to PvAVH53 or removal of the NLS peptides from PvAVH53 did not completely alter the subcellular localization; rather, these PvAVH53 mutants changed from being localized in the nucleus to being distributed in the nucleus and cytoplasm in both $V$. vinifera and N. benthamiana. However, PvAVH54804, with the same NES signal, was completely localized in the cytoplasm or distributed in only the nucleus with the NLS signal, demonstrating that the transport signals (namely, NLS and NES) were functional for protein transport; thus, PvAVH53 may have evolved other features that contribute to strong targeting to the plant cell nucleus, perhaps by interacting with endogenous host proteins. Moreover, all the modified effectors showed a significantly decreased ability to trigger cell death, indicating that the differences in localization may reflect the functional differences of the proteins, or to function properly based on the localization of the effectors. We identified VvImpas as targets of PvAVH53 using a yeast two-hybrid assay. As reported in previous studies, importin- $\alpha$ s exhibit three highly conserved structural domains: the importin-â-binding domain, ten armadillo (Arm) repeats for the binding of NLS-carrying proteins, and a C-terminal region that functions as a nuclear export factor binding site ${ }^{41-43}$. VvImp $\alpha 4$ contains nine tandem Arm motifs, including a C-terminal typical Arm motif, rather than ten arm repeats as previously reported $^{44}$. Consistent with previous studies, we found that PvAVH53 interacts with Arm repeats of VvImpa4, especially the C-terminal Arm, via its NLS sequence. Previous studies have revealed that transport factors are targeted by pathogen effectors; for example, the
P. infestans RXLR effector AVR1 targets the exocyst subunit Sec5 to disturb vesicle trafficking, potentially regulating plant immunity ${ }^{45}$. HaRxL106 from Hyaloperonospora arabidopsidis interacts with the importin- $\alpha$ Arm repeat domain via an $\mathrm{NLS}^{24}$. In rice, the bacterial pathogens Xanthomonas oryzae pv. oryzae (Xoo) and Xanthomonas oryzae pv. oryzicola (Xoc) target OsImp $\alpha 1 \mathrm{a}$ and OsImp $\alpha 1 \mathrm{~b}$ to facilitate infection $^{46}$. However, multiple imp- $\alpha$ homologs/isoforms exist in plants ${ }^{12}$, and these may associate with distinct groups of cargo proteins. The formation of cargo/importin$\alpha$ complexes is not always unique for effectors with NLS sequences. For example, in a study of interactions between importin- $\alpha$ and 83 effectors from Hpa and Pseudomonas syringae, the Hpa effector HaRxL106 was found to target several importin- $\alpha$ s (including MOS6, importin- $\alpha 1$, importin- $\alpha 2$ and importin- $\alpha 4)$, whereas the effector HaRxL445 interacted specifically with MOS6 ${ }^{24,47}$. Our study addressed interactions between the VvImpa4 homologs VvImp- $\alpha$ in $V$. vinifera and PvAVH53. Furthermore, there were an additional 11 RxLR-type effectors; the nine that were localized in only the nucleus interacted with VvImpa4, but the two non-nuclear effectors failed to target VvImpa4. These results implied that effectors containing the NLS localized in the plant nucleus and interacted with VvImpa4, but effectors lacking the NLS did not. Moreover, these interactions were specific and general simultaneously for nuclear-only effectors, and effectors with non-NLS signals may also have evolved other features that allow them to target the plant cell cytoplasm and the nucleus at the same time.

Phylogenetic analyses showed high conservation and homology in VvImpas and NbImpas, indicating that the function of NbImpas in $N$. benthamiana is likely to apply to VvImpos in $V$. vinifera. Our experiments using $\mathrm{Y} 2 \mathrm{H}$ and BiFC assays support the interaction between the orthologs NbImpa1/2 and PvAVH53. We used BiFC to show the interaction between PvAVH53 and Importinas (VvImpa4,

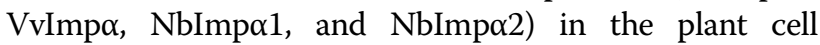
nucleus. Moreover, we took advantage of the virus-induced gene silencing (VIGS) and CRISPR/Cas13a systems to knock down the expression of two VvImportin- $\alpha 4$ homo-

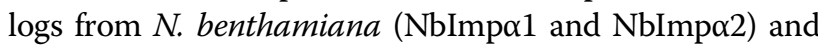
VvImp $\alpha / \alpha 4$ from grape. The CRISPR/Cas13a system driven by suitable promoters for dicot and monocot plants was introduced to target $\mathrm{RNA}^{37}$. It was previously reported that the nuclear localization of P. infestans Nuk6 and Nuk7, but not Nuk12, was affected in NbImpa1/2-silenced tobacco $^{12}$. Likewise, the PvAVH53 fluorescence-labelled protein was no longer localized in the nucleus in these NbImpa1/2-silenced tobacco and VvImpa4-silenced grape leaves, providing evidence that PvAVH53 relies on the importin $\alpha$-dependent nuclear import pathway to stabilize nuclear localization. Furthermore, transient expression of PvAVH53 and PvAVH54804 in NbImpo1/2-silenced 
tobacco showed the opposite effects: PvAVH53 failed to induce cell death, but PvAVH54804 triggered cell death. Moreover, reducing NbImp- $\alpha 1 / 2$ expression levels also increased the susceptibility to $P$. capsici, suggesting that NbImp- $\alpha 1 / 2$ may be directly or indirectly involved in transporting PvAVH53 to the nucleus and is required for immunity to $P$. capsici. At the same time, we used the CRISPR/Cas13a system to silence VvImp $\alpha / \alpha 4$ in grape and decrease the resistance to $P$. viticola. The results showed that VvImpos was required for the nuclear localization of PvAVH53 and was also required for overcoming P. viticola infection in grape. These results are consistent with a previous study showing that mutation of MOS6 (AtImportin- $\alpha 3$ ) promoted susceptibility to the oomycete plantpathogen Hyaloperonospora parasitica ${ }^{27}$. As previously reported, Importin- $\alpha$ s benefit infection by both viruses and bacteria, but the opposite is true for oomycetes. All the studies suggested that in nuclear transport trafficking, which is central to plant-pathogen interactions, Importin $\alpha$ has multiple function in plants and plays a significant role in plant defense and development. A previous study found that some proteins from pathogens are dependent on NbImp- $\alpha 1 / 2$ or close homologs for nuclear import, but some can target the nucleus independently of $\alpha$-Importins $^{12}$. Altogether, these results demonstrate that VvImpa4 or similar $\alpha$-Importins (such as NbImp- $\alpha 1 / 2$ ) are required for PvAVH53 to target the nucleus and induce cell death in $N$. benthamiana and increase susceptibility to $P$. capsici and P. viticola. However, importinos, for plant immunity, are a double-edged sword. On the one hand, they transport plant immune response-related proteins such as the late blight resistance protein R1, the shortened NLR protein TN13, and the polypolymerase PARP2 to contribute to immunity ${ }^{10,28,48}$. On the other hand, importin- $\alpha$ s also benefit pathogens, such as Pelargonium line pattern virus and Xanthomonas oryzae pv. oryzicola ${ }^{9,10,49}$.

In summary, we conclude that PvAVH53 depends on Importin- $\alpha$ s for transport to the nucleus in $V$. vinifera and $N$. benthamiana and that the canonical importin- $\alpha$ import pathway contributes to plant immunity or the pathogenicity of plant pathogens. To date, our understanding of how $P$. viticola effectors modulate host immunity remains limited. Future studies will focus on the targets of pathogen effectors in the host nucleus and the mechanism involved in the response to infection.

\section{Materials and methods Plasmid constructions}

The sequence and accession numbers for effector proteins are listed in Table S1. Effector genes, minus signal peptide sequences, were cloned from $P$. viticola (strain YL) genomic DNA using PCR and oligonucleotide primers, as listed in Table S2. Incorporation of NES (nuclear export signal) and NLS (nuclear import signal) sequences into the cloned effector genes was previously reported by $\mathrm{Du}$ et al, $2015^{19}$. The modified effector gene sequence was ligated into a derivative of the pCAMBIA2300 plasmid vector containing the green fluorescent protein (GFP) sequence. The oligonucleotide primers used in PCR-based cloning of IMPOR$T I N-\alpha$ homologs are listed in Table S2. For the gene silencing assay, a segment of NbImp $\alpha 1 / \mathrm{NbImp} \alpha 2$ was amplified from $N$. benthamiana cDNA using the primers Imp $\alpha 1 / 2-T R V 2-F$ and Impo1/2-TRV2-R and ligated into the plasmid vector pTRV2 $^{25}$. For CRISPR/Cas13a vector construction $^{37}$, the target sgRNA of VvImpo and VvImpa4 was designed using online software (http://crispr.dfci. harvard.edu/SSC/). Next, the forward and reverse primers of sgRNA were mixed equally at $98^{\circ} \mathrm{C}$ for $5 \mathrm{~min}$ and then placed in an ice bath for $5 \mathrm{~min}$. Finally, the products were ligated into the vector pCR11 described by Zhang Tong et al $(2019)^{48}$. The constructs were transformed into Escherichia coli strain Top 10 and subsequently cultured at $37^{\circ} \mathrm{C}$ on agar-solidified LB (Luria-Bertani) medium with screening antibiotics.

\section{Plant materials, fungal materials and inoculation}

Leaves of 4- to 5-week-old $N$. benthamiana and $V$. vinifera cv. Thompson Seedless were prepared for the following experiments. $N$. benthamiana plants were grown in a greenhouse for use in both Agrobacterium-mediated transient gene expression assays and $P$. capsici infection assays. Inoculation of leaves with $P$. capsici and $P$. viticola was carried out as described previously ${ }^{22,33}$. Briefly, $P$. capsici cultures were maintained on $10 \%$ V8 juice agar medium at $25^{\circ} \mathrm{C}$ in the dark. Sporulation was induced on $10 \%$ V8 juice mix medium (solid and liquid) by incubation at $4{ }^{\circ} \mathrm{C}$. Zoospores were eluted in sterile water, and the concentration was adjusted to $1.0 \times 10^{4} \mathrm{~mL}^{-1}$. Detached leaves were inoculated with a $10 \mu \mathrm{L}$ droplet of zoospore suspension and incubated under high humidity at $25^{\circ} \mathrm{C}$ in the dark. Lesion areas were measured 2 days after infection. One-centimeter diameter leaf discs of the susceptible $V$. vinifera cv. Thompson Seedless were inoculated with $20 \mu \mathrm{L}$ of a $P$. viticola sporangial suspension $\left(5 \times 10^{4}\right.$ sporangia/ $\mathrm{mL})$ for 1 day, and then, the remaining suspension was removed and placed on wet sterile filter paper in a growth chamber at $22^{\circ} \mathrm{C}$.

\section{Transient transformation expression assays}

Plasmid constructs were introduced into A. tumefaciens strain GV3101 by electroporation, and transformants were selected on solidified LB medium containing kanamycin $(50 \mu \mathrm{g} / \mathrm{mL})$, gentamycin $(50 \mu \mathrm{g} / \mathrm{mL})$ and rifamycin $(50 \mu \mathrm{g} / \mathrm{mL})$. Recombinant Agrobacterium strains were grown in liquid $\mathrm{LB}$ medium at $28^{\circ} \mathrm{C}$ with shaking at $200 \mathrm{rpm}$. After $20 \mathrm{~h}$ of growth, cells were collected by centrifugation at $5000 \times \mathrm{g}$ for $3 \mathrm{~min}$, washed twice with $500 \mathrm{mM} \mathrm{MgCl} 2$, and then resuspended in infiltration 
medium (10 mM $\mathrm{MgCl}_{2}, 10 \mathrm{mM}$ MES (pH 5.7), $200 \mu \mathrm{M}$ acetosyringone) to an $\mathrm{OD}_{600}$ of 0.4 . Cells were then maintained for $3 \mathrm{~h}$ at $28^{\circ} \mathrm{C}$ in the dark prior to infiltra$\operatorname{tion}^{50}$. Infiltration was performed with leaves of $N$. benthamiana and $V$. vinifera using a 1-mL syringe, and plants were further grown in controlled conditions under a photoperiod of 16 -h light at $25^{\circ} \mathrm{C}$ and 8 -h dark at $18{ }^{\circ} \mathrm{C}$. All experiments were performed at least three times.

\section{TRV-induced gene silencing}

Analysis of gene function by virus-induced gene silencing (VIGS) was performed by using a tobacco rattle virus (TRV)-based vector ${ }^{35,51}$. VIGS constructs (pTRV2:GFP ${ }^{52}$ and pTRV2:NbImpa1/NbImp 2 2) were introduced into $A$. tumefaciens strain GV3101 by electrotransformation.

Bacterial cultures were grown to $\mathrm{OD}_{600}=0.1$ and then combined at a 1:1 ratio and injected into fully expanded leaves of 2-week-old $N$. benthamiana plants. Plants subjected to infiltration were grown for 2-3 weeks in a greenhouse under a daily regime of 16 -h light at $22^{\circ} \mathrm{C}$ and 8 -h dark at $18{ }^{\circ} \mathrm{C}$. Oligonucleotide primer sequences used for detecting the silenced gene by quantitative RT-PCR are given in Supplemental Table 2. The NbEF1 $\alpha$ gene was used as an internal reference ${ }^{29}$.

\section{Bimolecular fluorescence complementation and confocal imaging}

Analyses of intracellular protein interactions and colocalization using bimolecular fluorescence complementation $(\mathrm{BiFC})^{53}$ assays were performed by using protoplasts derived from healthy and fully expanded tobacco leaves or callus tissue prepared from cv. Thompson Seedless. Protoplast preparation and polyethylene glycol (PEG)-mediated transformation of the protoplasts were carried out as previously described ${ }^{54}$. PvAVH53 and VvImp $\alpha$ gene fragments were cloned into pUC-SPYNE and pUC-SPYCE, respectively, to create PvAVH53-YN and VvImpas-YC. The PvAVH53-YN and VvImpas-YC constructs were cotransformed into protoplasts. Empty pUC-SPYNE and pUCSPYCE vectors were used as negative controls. Protoplasts were incubated for $20-24 \mathrm{~h}$ under weak lighting at $25^{\circ} \mathrm{C}$ prior to imaging by confocal microscopy (Germany, Leica TCS SP8). The wavelengths used for excitation of GFP, YFP and mCherry were 488,630 , and $561 \mathrm{~nm}$, respectively.

\section{Protein extraction and western blotting}

To detect the expression of recombinant proteins, protein extraction and western blotting were carried out as described in Chen et al. (2020) ${ }^{33}$. In brief, proteins extracted by using an extraction buffer were added to $1 \times$ loading buffer and incubated in a boiling water bath for $5 \mathrm{~min}$. The supernatant was loaded on a 10\% SDS-PAGE gel, and proteins were transferred to a PVDF membrane using a Trans-Blot cell (America, Biorad Trans-Blot SD). The membrane was incubated with $5 \%$ nonfat dry milk in TBST (20 mM Tris- $\mathrm{HCl}, 150 \mathrm{mM} \mathrm{NaCl}, 0.05 \%$ Tween-20) for $3 \mathrm{~h}$ at room temperature. Mouse anti-GFP monoclonal antibodies (ABclonal) were then added to the buffer at a ratio of 1:5000, and the membrane was shaken slowly overnight at $4^{\circ} \mathrm{C}$ and then washed in TBST. Goat anti-mouse IRDye $800 \mathrm{CW}$ was then added at a ratio of $1: 10,000$, and the membrane was shaken for an additional $1 \mathrm{~h}$. The membrane was washed five times in TBST for 5 min each time and then visualized using ChemiDocTM XRS + software.

\section{Bioinformatics and sequence analysis}

NLS signal sequences were predicted using cNLS Mapper (http://nls-mapper.iab.keio.ac.jp/cgi-bin/NLS_Mapper_form. cgi\#topennewwindow). The domain organization of VvImpa4 was analyzed using Pfam (http://pfam.xfam.org/).

Protein sequence alignment was performed by using DNAMAN BLAST. The phylogenetic tree was constructed using MEGA5 with the neighbor-joining method, 1000 replicates, and the pairwise deletion option.

\section{Visualization of cell survival by GUS staining}

For evaluation of $N$. benthamiana cell survival following coinfiltration with pBI121::GUS and RxLR53 or RxLR54804, histochemical analysis of GUS activity was carried out 4 days after infiltration as described previously ${ }^{55}$. The GFPeffector fusion protein, GFP (as a negative control) and PGR107-NotI::INF1 (as a positive control) were each coexpressed with PBI121::GUS in N. benthamiana leaves using agroinfiltration. Photographs were taken $4 \mathrm{~d}$ post infiltration.

\section{Trypan blue staining and aniline blue staining}

For trypan blue staining, $N$. benthamiana leaves were immersed in trypan blue solution ( $10 \mathrm{~g}$ of phenol, $10 \mathrm{ml}$ of glycerol, $10 \mathrm{ml}$ of distilled water, and $0.02 \mathrm{~g}$ of trypan blue), boiled in a water bath for $2 \mathrm{~min}$, incubated for $1 \mathrm{~d}$ at room temperature, and then destained in chloral hydrate solution ( $250 \mathrm{~g}$ of chloral hydrate per $1 \mathrm{ml}$ of water) for 1 $\mathrm{d}$ followed by $95 \%$ ethyl alcohol for $2 \mathrm{~d}$. Samples were then maintained in $75 \%$ ethyl alcohol prior to being photographed. To detect the development of $P$. viticola infection in grape leaves, the leaves were decolorized using 95\% alcohol in boiled water for $5 \mathrm{~min}$, and alcohol was removed and incubated in $0.05 \%$ aniline blue dissolved in $0.067 \mathrm{M} \mathrm{K}_{2} \mathrm{HPO}_{4}(\mathrm{pH}=9-10)$ for one night. The photographs were obtained by a fluorescence microscope (Olympus bx-51). The wavelengths used for excitation were $400-440 \mathrm{~nm}$ (blue/purple $)^{56,57}$.

\section{Measurement of ion leakage}

Following infiltration, leaf discs $(1 \mathrm{~cm}$ diameter) were immersed in $5 \mathrm{ml}$ of distilled water in a $50-\mathrm{ml}$ sterile centrifuge tube with gentle shaking at $50 \mathrm{rpm}$ for $3 \mathrm{~h}$ at 
room temperature. Ion leakage was measured with a conductivity meter (DDS-307, LeiCi, Shanghai, China). Total ion leakage was measured after incubation in a boiling water bath for $25 \mathrm{~min}$. The results are expressed as a percentage of total ion leakage.

\section{Yeast two-hybrid screening and assays}

To screen for effector-interacting proteins, a cDNA library was prepared from the susceptible $V$. vinifera cultivar 'Pinot Noir' inoculated with P. viticola. Screening was carried out using the Yeastmaker ${ }^{\mathrm{TM}}$ Yeast Transformation System 2 (Clontech) according to the manufacturer's protocol. For analyses of effector-importin- $\alpha$ interactions, the $P$. viticola effector sequence was inserted into the plasmid pGBK-T7 (BD), and the importin- $\alpha$ sequence was ligated into the plasmid pGAD-T7 (AD).

\section{Acknowledgements}

We thank the staff of the laboratory for their technical assistance and Professor Zhang Tong for offering the vector pCR11. We thank Prof. Steven van Nocker for critically reading the manuscript and for his helpful suggestions. This work was supported by the National Natural Science Foundation of China (grant No. $31872054,31672115,31471844)$, the Program for "Special Fund for Agroscientific Research in the Public Interest" (grant No. 201203075-08), the National Innovation Experimental Program for Undergraduates from Northwest A\&F University, China (grant No. 201610712008), and the Innovation Fund for Young Talents in Fujian Academy of Agricultural Sciences (YC2016-2).

\section{Author details \\ ${ }^{1}$ State Key Laboratory of Crop Stress Biology in Arid Areas (Northwest A\&F University), Yangling, Shaanxi, P.R. China. ${ }^{2}$ College of Horticulture, Northwest A\&F University, Yangling, Shaanxi, P.R. China. ${ }^{3}$ Key Laboratory of Horticultural Plant Biology and Germplasm Innovation in Northwest China, Ministry of Agriculture, Yangling, Shaanxi, P.R. China. ${ }^{4}$ Fruit Research Institute, Fujian Academy of Agricultural Sciences, 350013 Fuzhou, Fujian, China}

\section{Author contributions}

C.T.T. and X.Y. designed the research; P.J. performed all gene cloning and plasmid vector construction. C.T.T. and P.J. performed the analyses of subcellular localization and protein interactions. L.M.J. and Y.X. prepared the plant materials. C.T.T. drafted the manuscript, and W.Y.J. and L.Y. modified the language. All authors have read and approved the final manuscript.

\section{Conflict of interest}

The authors declare that they have no conflict of interest.

Supplementary information The online version contains supplementary material available at https://doi.org/10.1038/s41438-021-00482-6.

Received: 27 July 2020 Revised: 3 December 2020 Accepted: 12 December 2020

Published online: 01 March 2021

\section{References}

1. Kamoun, S. A catalogue of the effector secretome of plant pathogenic oomycetes. Annu. Rev. Phytopathol. 44, 41-60 (2006).

2. Kamoun, S. Groovy times: filamentous pathogen effectors revealed. Curr. Opin. Plant Biol. 10, 358-365 (2007).

3. Birch, P. R. J., Armstrong, M., Bos, J., Boevink, P. \& Sophien, K. Towards understanding the virulence functions of RXLR effectors of the oomycete plant pathogen Phytophthora infestans. J. Exp. Bot. 60 , 1133-1140 (2009).
4. Bozkurt, T. O., Schornack, S., Banfield, M. J. \& Kamoun, S. Oomycetes, effectors, and all that jazz. Curr. Opin. Plant Biol. 15, 483-492 (2012).

5. Boch, J. \& Bonas, U. Xanthomonas AvrBs3 family-type III effectors: discovery and function. Annu. Rev. Phytopathol. 48, 419 (2010).

6. Caillaud, M. C., Piquerez, S. J. M., Fabro, G., Steinbrenner, J. \& Jones, J. D. G. Subcellular localization of the Hpa RxLR effector repertoire identifies a tonoplast-associated protein HaRxL17 that confers enhanced plant susceptibility. Plant J. 69, 252-265 (2011).

7. Wang, X. et al. A host KH RNA-binding protein is a susceptibility factor targeted by an RXLR effector to promote late blight disease. Mol. Plant $\mathbf{8}$, 1385-1395 (2015).

8. Boevink, P. C. et al. A Phytophthora infestans RXLR effector targets plant PP1C isoforms that promote late blight disease. Nat. Commun. 7, 10311 (2016).

9. Wang, H., Ren, Y., Zhou, J., Du, J. \& Xie, C. The cell death triggered by the nuclear localized RxLR effector PITG_22798 from Phytophthora infestans is suppressed by the effector AVR3b. Int. J. Mol. Sci. 18, 409 (2017).

10. Du, Y., Berg, J., Govers, F. \& Bouwmeester, K. Immune activation mediated by the late blight resistance protein $\mathrm{R} 1$ requires nuclear localization of $\mathrm{R} 1$ and the effector AVR1. N. Phytol. 207, 735-747 (2015).

11. Cassimeris, L., Plopper, G. \& Lingappa, V. R. Lewin's CELLS. 2th ed. Sulbury: Jones and Bartlett Publishers (2011).

12. Kanneganti, T. D. et al. A functional genetic assay for nuclear trafficking in plants. Plant J. 50, 149-158 (2007).

13. Gorlich, D. \& Mattaj, I. W. Nucleocytoplasmic transport. Science 271, 1513-1519 (1996).

14. Lange, A. et al. Classical nuclear localization signals: definition, function, and interaction with importin a. J. Biol. Chem. 282, 5101-5105 (2007).

15. Fahrenkrog, B. \& Aebi, U. The nuclear pore complex: nucleocytoplasmic transport and beyond. Nature Reviews Molecular Cell Biology 4, 757 (2003).

16. Stewart, M. Molecular mechanism of the nuclear protein import cycle. Nat Rev. Mol. Cell. Biol. 8, 195-208 (2007).

17. Matsuura, Y. \& Stewart, M. Structural basis for the assembly of a nuclear export complex. Nature 432, 872-877 (2004)

18. Oka, M. \& Yoneda, Y. Importin a: functions as a nuclear transport factor and beyond. Proc. Jpn. Acad. 94, 259-274 (2018).

19. Robbins, J., Dilwortht, S. M., Laskey, R. A. \& Dingwall, C. Two interdependent basic domains in nucleoplasmin nuclear targeting sequence: Identification of a class of bipartite nuclear targeting sequence. Cell $\mathbf{6 4}$, 615-623 (1991).

20. Chang, C. W., Couñago, R. L. M., Williams, S. J., Bodén, M. \& Kobe, B. Crystal structure of rice importin- and structural basis of its interaction with plantspecific nuclear localization signals. Plant Cell. 24, 5074-5088 (2012).

21. Schornack, S. et al. Ancient class of translocated oomycete effectors targets the host nucleus. Proc. Natl Acad. Sci. USA 107, 17421-17426 (2010).

22. Song, T. et al. An oomycete CRN effector reprograms expression of plant HSP genes by targeting their promoters. PLoS Pathog. 11, e1005348 (2015).

23. Xiang, J. et al. A candidate RxLR effector from Plasmopara viticola can elicit immune responses in Nicotiana benthamiana. BMC Plant Biol. 17, 75 (2017).

24. Wirthmueller, L. et al. Probing formation of cargo/importin-a transport complexes in plant cells using a pathogen effector. Plant J. 81, 40-52 (2015).

25. Bai, $X$. et al. AY-WB phytoplasma secretes a protein that targets plant cell nuclei. Mol. Plant Microbe Interact. 22, 18-30 (2009).

26. Sugio, A., Maclean, A. M. \& Hogenhout, S. A. The small phytoplasma virulence effector SAP11 contains distinct domains required for nuclear targeting and CIN-TCP binding and destabilization. N. Phytol. 202, 838-848 (2014).

27. Palma, K. Zhang, Y. \& Li, X. An importin alpha homolog, MOS6, plays an important role in plant innate immunity. Current Biology. 15, 1129-1135 (2005).

28. Roth, C. et al. The truncated NLR protein TIR-NBS13 is a MOS6/MPORTIN-a3 interaction partner and required for plant immunity. Plant J. 92, 808-821 (2017).

29. Yin, X. et al. The nuclear-localized RxLR effector PvAvh74 from Plasmopara viticola induces cell death and immunity responses in Nicotiana benthamiana. Front. Microbiol. https://doi.org/10.3389/fmicb.2019.01531 (2019).

30. Kinkema, M., Weihua, F. \& Xinnian, D. Nuclear localization of NPR1 is required for activation of PR gene expression. Plant Cell 12, 2339-2350 (2000).

31. Mou, Z., Weihua, F. \& Xinnian, D. Inducers of plant systemic acquired resistance regulate NPR1 function through redox changes. Cell 113, 0-944 (2003).

32. Zhang, Y. \& Li, X. A putative nucleoporin 96 is required for both basal defense and constitutive resistance responses mediated by suppressor of npr1-1, constitutive 1. Plant Cell 17, 1306-1316 (2005). 
33. Chen, T. et al. Insight into function and subcellular localization of Plasmopara viticola putative RxLR effectors. Front. Microbiol. 11, 692 (2020).

34. Mittler, R. et al. Transgenic tobacco plants with reduced capability to detoxify reactive oxygen intermediates are hyperresponsive to pathogen infection. Proc. Natl Acad. Sci. USA 96, 14165-14170 (1999).

35. Ratcliff, F., Ana Montserrat, M. H. \& Baulcombe, D. C. Technical advance: tobacco rattle virus as a vector for analysis of gene function by silencing. Plant J. 25, 237-245 (2008).

36. Abudayyeh, O. O. et al. C2C2 is a single-component programmable RNAguided RNA-targeting CRISPR effector. Science 353, aaf5573 (2016).

37. Zhang, T. et al. Establishing CRISPR/Cas13a immune system conferring RNA virus resistance in both dicot and monocot plants. Plant Biotechnol. J. 17, 1185-1187 (2019).

38. Ketelaar, T. et al. Positioning of nuclei in Arabidopsis root hairs. The Plant Cell 14, 2941-2955 (2002).

39. Iwabuchi, K., Minamino, R. \& Takagi, S. Actin reorganization underlies phototropin-dependent positioning of nuclei in Arabidopsis leaf cells. Plant Physiol. 152, 1309-1319 (2010).

40. King, S. R. et al. Phytophthora infestans RXLR effector PexRD2 interacts with host MAPKKK epsilon to suppress plant immune signaling. Plant Cell 26, 1345-1359 (2014).

41. Kutay, U., Bischoff, F. R., Kostka, S., Kraft, R. \& Görlich, D. Export of importina From the nucleus is mediated by a specific nuclear transport factor. Cell $\mathbf{6 6}$, 1061-1071 (1997).

42. Herold, A., Truant, R., Wiegand, H. \& Cullen, B. R. Determination of the functional domain organization of the importin alpha nuclear import factor. J. Cell Biol. 143, 309-318 (1998).

43. Miyamoto, Y., Peter, R. B., Gary, R. H. \& Kate, L. L. Regulated nucleocytoplasmic transport during gametogenesis. Biochim. Biophys. Acta. 1819, 616-630 (2012)

44. Goldfarb, D. S. \& Corbett, A. H. et al. Importin a: a multipurpose nucleartransport receptor.Trends Cell Biol 14, 505-514 (2004).

45. Du, Y., Mpina, M., Birch, P., Bouwmeester, K. \& Govers, F. Phytophthora infestans RXLR effector AVR1 interacts with exocyst component Sec5 to manipulate plant immunity. Plant Physiol. 169, 1975-1990 (2015)
46. Hui, S. et al. TALE-carrying bacterial pathogens trap host nuclear import receptors for facilitating infection of rice. Mol. Plant Pathol. 20, 519-532 (2019).

47. Mukhtar, M. S. et al. Independently evolved virulence effectors converge onto hubs in a plant immune system network. Science 333, 596-601 (2011).

48. Chen, C., Masi, R. D., Lintermann, R. \& Wirthmueller, L. Nuclear import of Arabidopsis poly(ADP-Ribose) polymerase 2 is mediated by importin-a and a nuclear localization sequence located between the predicted SAP domains. Front. Plant Sci. 9, 1581 (2018).

49. Pérez-Cañamás, M. \& Hernández, C. New insights into the nucleolar localization of a plant RNA virus-encoded protein that acts in both RNA packaging and RNA silencing suppression: involvement of importins alpha and relevance for viral infection. Mol. Plant Microbe Interact. 31, 1134-1144 (2018).

50. Van der Hoorn, R. A., Laurent, F., Roth, R. \& De Wit, P. J. Agroinfiltration is a versatile tool that facilitates comparative analyses of Avr9/Cf-9-induced and Avr4/Cf-4-induced necrosis. Mol. Plant Microbe Interact. 13, 439-446 (2000)

51. Baulcombe, D. C. Fast forward genetics based on virus-induced gene silencing. Curr. Opin. Plant Biol. 2, 109-113 (1999).

52. McLellan, $H$. et al. An RxLR effector from Phytophthora infestans prevents relocalisation of two plant NAC transcription factors from the endoplasmic reticulum to the nucleus. PLoS Pathog. 9, e1003670 (2013).

53. Martin, K. et al. Transient expression in Nicotiana benthamiana fluorescent marker lines provides enhanced definition of protein localization, movement and interactions in planta. Plant J. 59, 150-162 (2009).

54. Yoo, S. D., Cho, Y. H. \& Sheen, J. Arabidopsis mesophyll protoplasts: a versatile cell system for transient gene expression analysis. Nat. Protoc. 2, 1565-1572 (2007).

55. Xu, W. et al. Expression pattern, genomic structure, and promoter analysis of the gene encoding stilbene synthase from Chinese wild Vitis pseudoreticulata. J. Exp. Bot. 62, 2745-2761 (2011).

56. Liu, R. et al. Histological responses to downy mildew in resistant and susceptible grapevines. Protoplasma 252, 259-270 (2015).

57. Li, M. et al. CRISPR/Cas9-mediated VVPR4b editing decreases downy mildew resistance in grapevine (Vitis vinifera L.). Hortic. Res. 7, 149 (2020). 